\title{
The Development of the General Computer Control System Based on Multi- functional Triaxial Apparatus
}

\author{
Chuan Huang ${ }^{1, a^{*}}$, Haitao $\mathrm{Wu}^{1, \mathrm{~b}}$ and Chunmin Yuan ${ }^{1, \mathrm{c}}$ \\ ${ }^{1}$ School of Mathematic \& Computer Science. Wuhan Polytechnic University, Wuhan China \\ a949314445@qq.com, b691650480@ qq.com, ${ }^{\mathrm{c}} 87746570 @ q q . c o m$ \\ *The corresponding author
}

Keyword: Triaxial apparatus; Digital image measurement; Measure \& control system; Data acquiring.

\begin{abstract}
The triaxial apparatus is a typical application of electromechanical technology in soil mechanics measurement. We make an overall analysis of function requirements of the triaxial apparatus and propose a system solution based on up/lower computer system architecture. At last, we give a experimental case at end of paper.
\end{abstract}

\section{Introduction}

Triaxial apparatus was invented by Casagrande at 1930s, it has been the main method of determination of soil shear strength. The paper's main work is in using computer control system to implement the automatic collection, storage and processing data. And the paper implements a general hardware and software architecture of triaxial apparatus.

\section{System Architecture}

As one of best tools in soil test, triaxial apparatus has been a mature technology. This paper developed a multi-fuctional triaxial apparatus which has a higher level of automation and could reduce experimenter burden. The most effective methods was to build the architecture to up-host system, down-host system and the digital image measurement system:

Up-host system was based by PC, it include human-computer interface module, test logical control module, etc.

Down-host system was based by embedded system, it include data sampling module, stepper motor control module, etc.

Can-bus system was used to connect up-host and down-host

Digital image measurement system was used to do non-contact measurement of deformation process

System framework is as follows: 


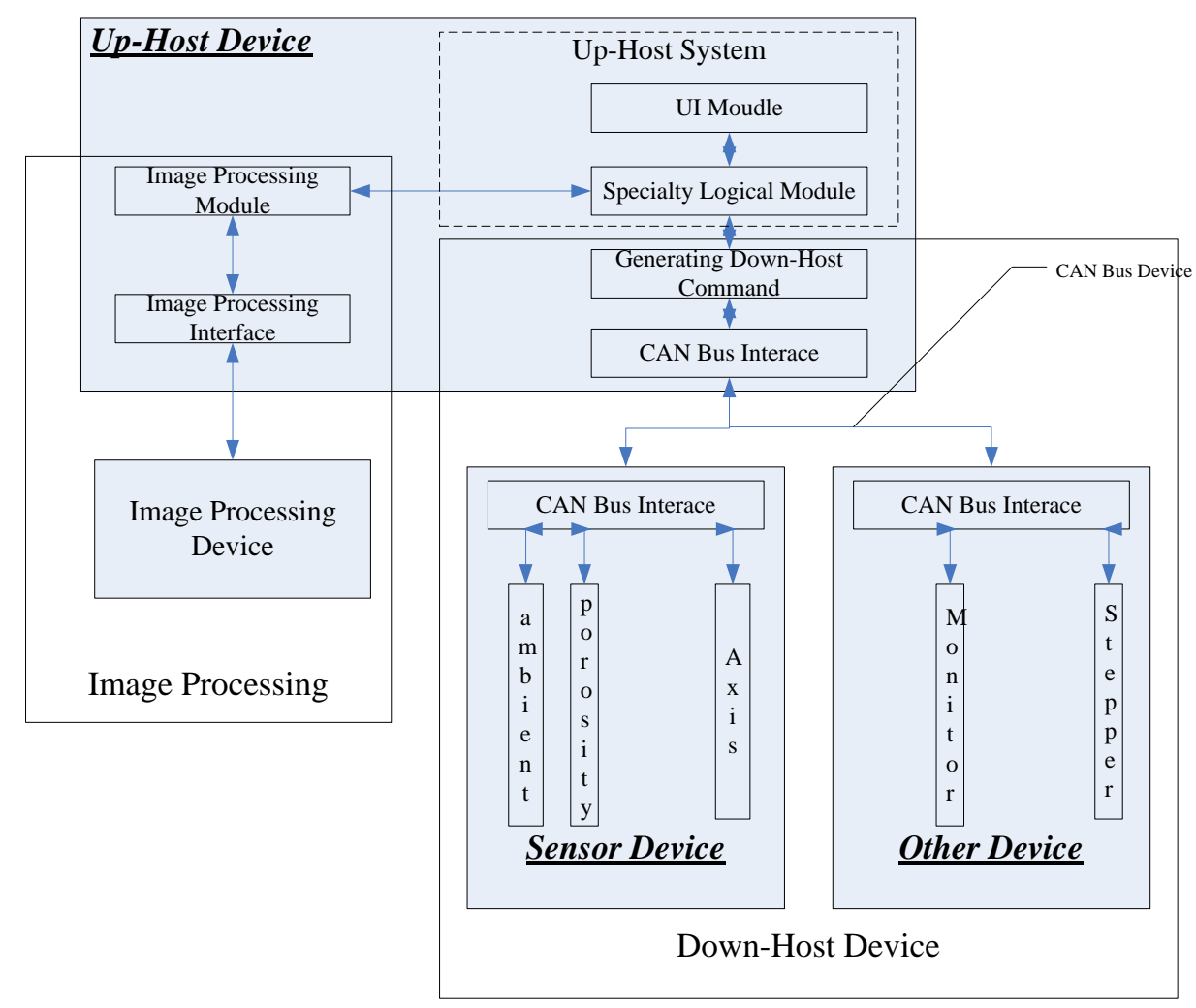

Figure 1. $\quad$ system framework and function modules

\section{Up-host and Down-host Explanations}

Different from embeded system, PC system has enough resource. So we could put calculation module and display calculation in it. There are three layers in up-host system:

UI module: user input and result output in display;

Specialty logic module: system kernel module (all other modules connect with this module): 1) send input command to down-host; 2) active by information from down-host; 3) active by information from digital image measurement;

Transformation module: 1) up-host invokes the interface to build message and send to down-host; 2) down-host receive the message, analysis the message and active by the message.

By those common layers, if the transformation protocol did not change, other apparatus can use same modules in the future except display module/specialty logic module. For the convenience of developing common architecture, the system puts display module/specialty logic module in the up-host system. Architecture is as follows 


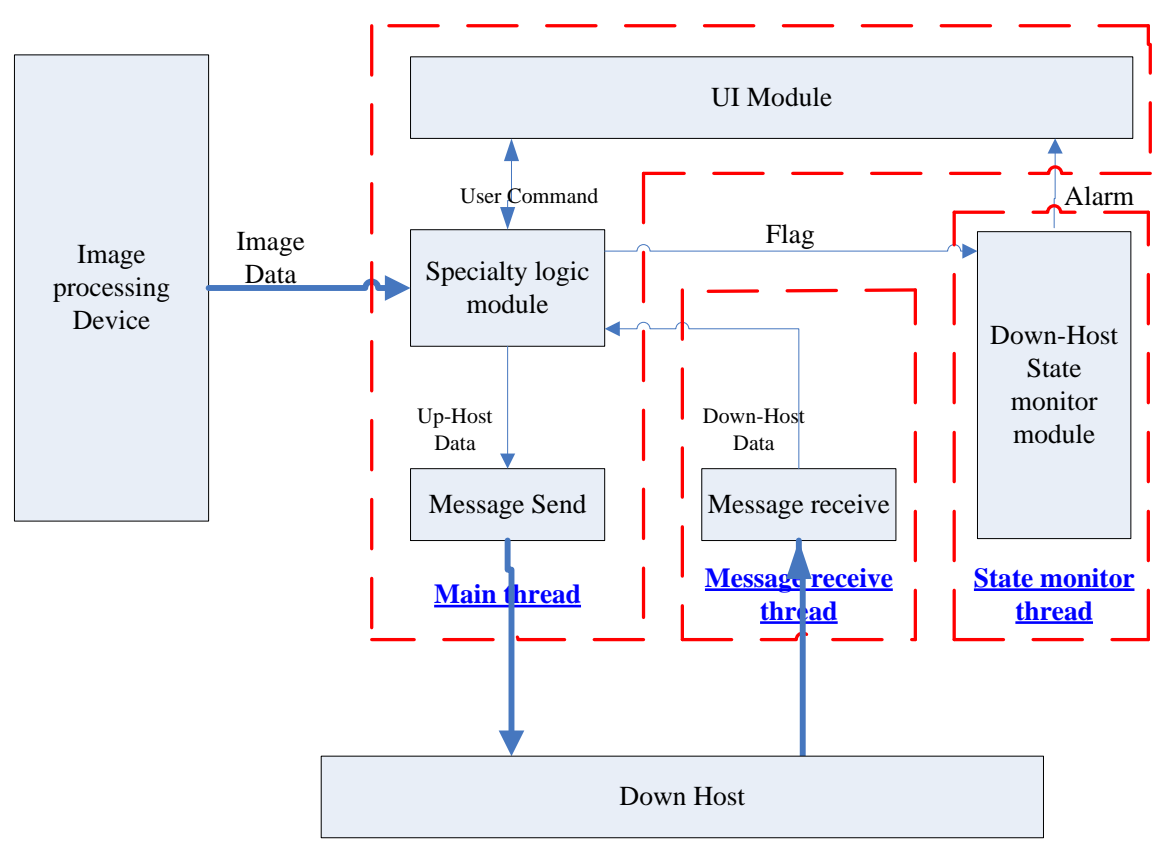

Figure 2. Up-host common logic process

In Fig. 2, up-host implement by multi-thread. One dashed box means a thread and one solid box means a function module, the arrow means data flow.

Main thread: Include UI module, specialty logic module and message send module. The kernel module is the specialty logic module, which connect to image-measure module, message receive module and state monitor module.

Message receiving thread: which receive message from CAN-bus. Message receive module and message send module is asynchronous.

State monitor thread: which send heartbeat packets on time to determine the state of down-host. So we need a independent thread.

The tasks of down-host get the data from sensors and control the stepper motor in real time. Because there are only a few device to control (3 stepper motors and 4 sensors), we choice EPC2903 as kernel module of embedded system, 8208BEH as data acquisition module, 8016A as stepper motor control module.

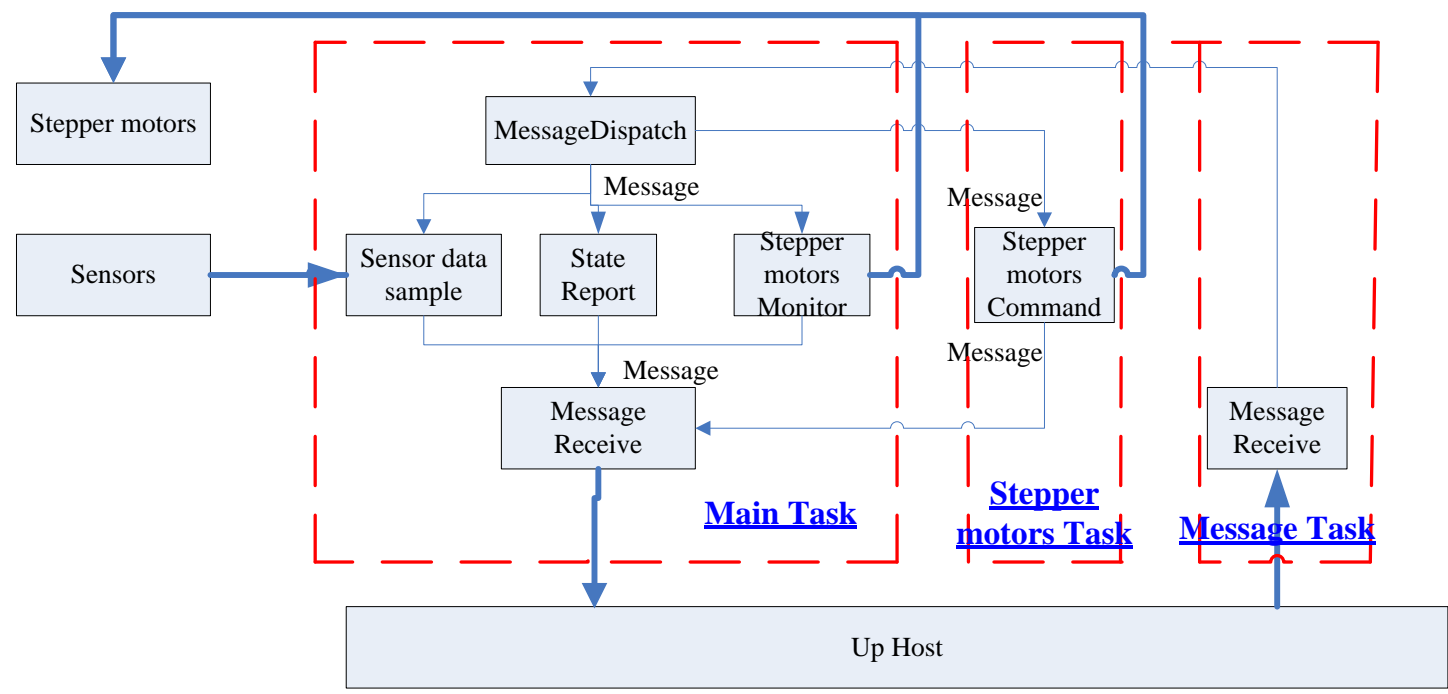

Figure 3. Architecture of down-host 
Saming as up-host, the down-host based on multi-tasks system to simplify logic process. One dashed box means a task and one solid box means a function module, the arrow means data flow:

Main task: It includes message-dispatch module, sensor data acquisition module, state monitor and report module. All task run on time.

Stepper motors control task: It includes stepper motors command transform module, which receives up-host command and send the command to stepper motors.

Message receive task: It receive the message from the up-host.

In the test, there need to share and transfer information in real-time. So a well communication bus is very important in the system. There are many communication bus, such as RS232, RS485, CAN, HART, PROFIUS, etc. The CAN bus has high reliability, good ability of error detection, which has been widely used in the computer control system in the car and kinds of industrial environment.System use USBCAN2 flexible interface card, which accord with CAN2.0A/B specification, support any baud rate between $5 \mathrm{Kbps} \sim 1 \mathrm{Mbps}$. The CAN message protocol based on iCAN protocol stack.

\section{System of Digital Image Processing}

The conventional measurement in triaxial apparatus adopt local displacement sensor. But if we use few sensor, we can not have enough data; if we use many sensor, sensor itself would affect sample. The DIP(Digital Image Precessing) is widely used and is in rapid development of technology, which characteristic is high accuracy, non-contact, adapting to the large deformation, undisturbing sample. The DIP algorithm in program based on OpenCV lib library. The measurement-processing is as fellow:

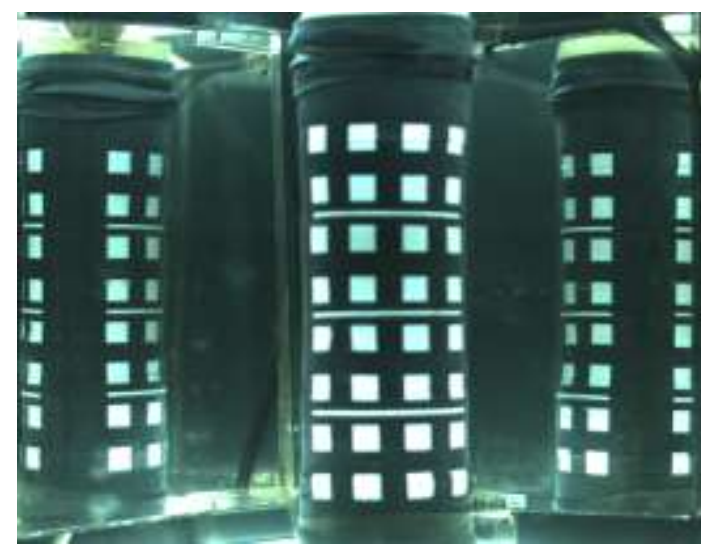

Figure 4. Original Image

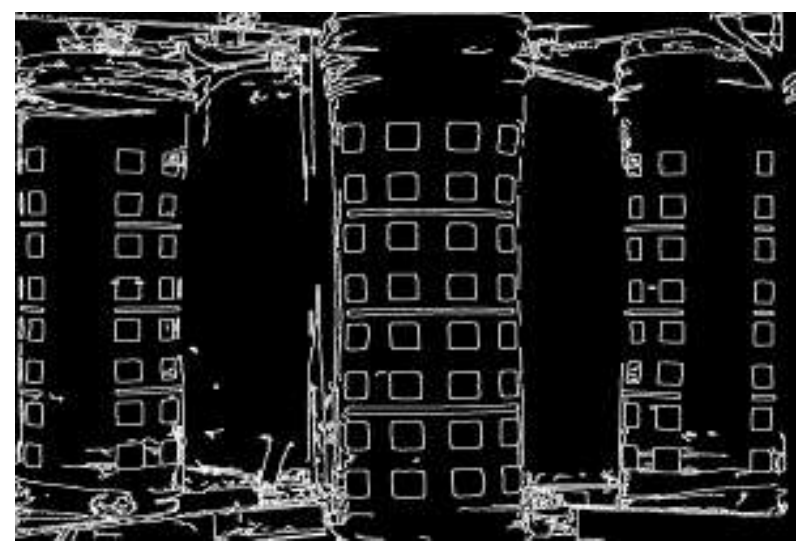

Figure 5. After canny edge detection

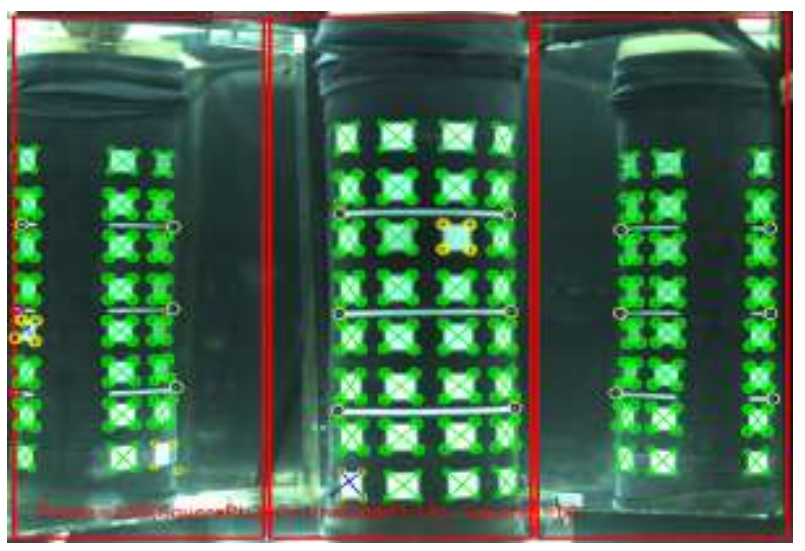

Figure 6. Feature points to capture 


\section{Summary}

The system implement a general computer control system not only on the multi-functional triaxial apparatus but also on other soil apparatus such as plane strain apparatus, unsaturation triaxial apparatus, etc. In the system, we use DIP technique in measurement, which could improve accuracy and save cost. The fellow figure is the sample after the test, the whole test could finish automatically.

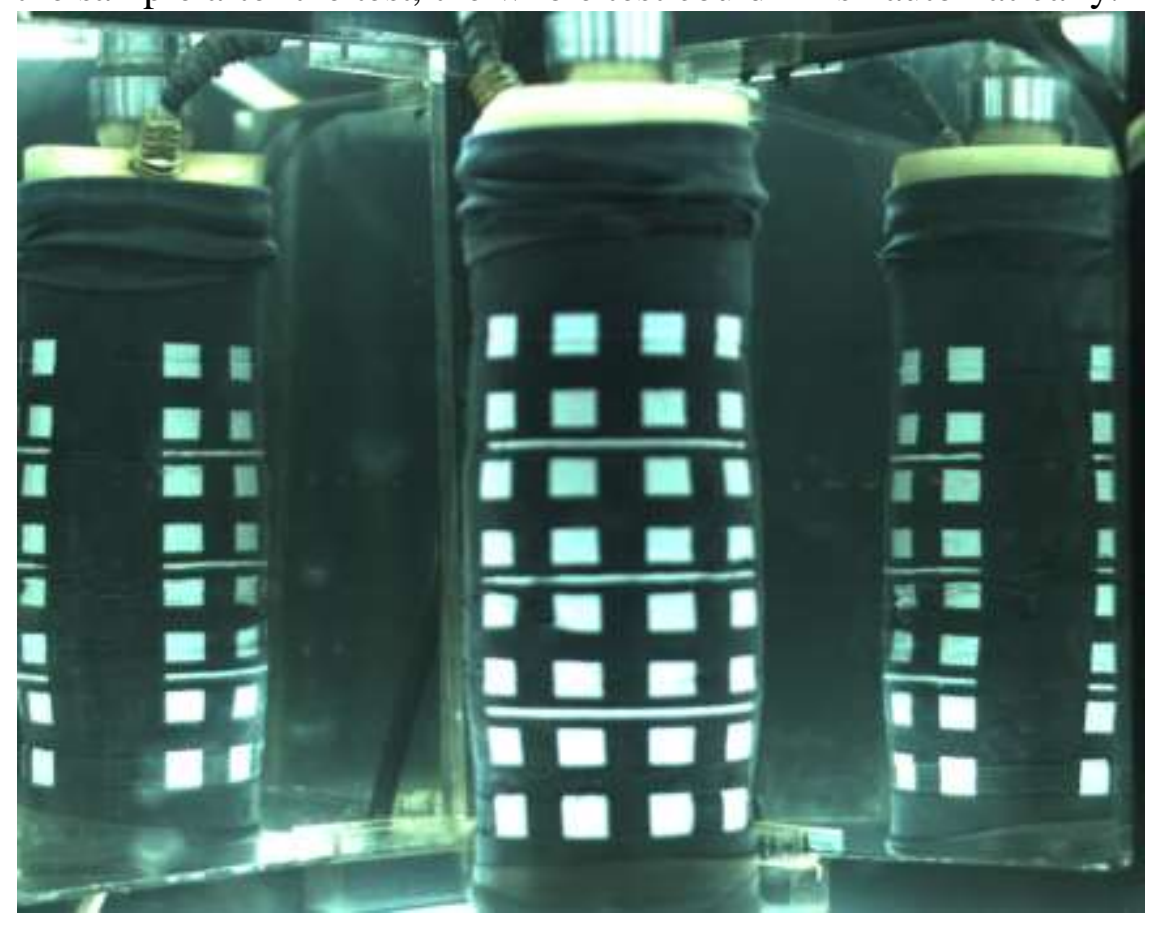

Figure 7. Sample after test

\section{Acknowledgement}

This work was supported by the Natural Science Foundation of Hubei Province of China under Grant No.2016CFB470.

\section{References}

[1] Raschke S A,Hryciw R D.Grain-size distribution of granular soils by computer vision.Geotechnical Testing Journal,1997,20(4):433-442.

[2] Bressani L A. External Measurement of Axial Strain in the Triaxial Test.Geotechnical Testing Journal,2005,18(2):226-240.

[3] Bishop A W, Henkel D F. The measurement of soil properties in the triaxial test[M]. London: Edward Amold Ltd, 1969.

[4] Garga V K,Zhang H. Volume changes during undrained triaxial tests on sands.Canadian Geotechnical Journal,2007,34(5):762-772.

[5] Fang L and Au O C. Subpixel-based image down-sampling with min-max directional error for stripe display[J]. IEEE Journal of Selected Topics in Signal Processing, 2015, 5(2):240-251.

[6] Kim Jun-seong and Kim Chang-su. A filter design algorithm for subpixel rendering on matrix displays[C]. European Signal Processing Conference, Poznan, Poland, 2009: 1487-1491.

[7] Fang L, Au O C, Yang Y, et al.. A new adaptive subpixelbased downsampling scheme using edge detection[C]. IEEE International Symposium on Circuits and Systems, Taipei,2016: 3194-3197 